\title{
Hemophilia therapy: the future has begun
}

\section{Pier Mannuccio Mannucci}

Fondazione IRCCS Ca' Granda Ospedale Maggiore Policlinico, Angelo Bianchi Bonomi Hemophilia and Thrombosis Center, Milan, Italy

\section{ABSTRACT}

T he success story of hemophilia care first began in the 1970s, when the availability of plasma-derived concentrates of coagulation factor VIII (FVIII) and factor IX (FIX) provided efficacious treatment of bleeding in patients with hemophilia A and B. This positive scenario was consolidated in terms of greater safety and availability in the 1990s, when the first recombinant coagulation factors were produced. This meant that, instead of only treating episodic bleeding events, prophylaxis regimens could be implemented as a preventive measure. Following the demonstration of its superiority in the frame of two randomized clinical trials, prophylaxis became evidence-based standard of care. In highincome countries, these achievements have led to a patients' life expectancy being extended to close to that of the general male population. Alongside this, the last decade has witnessed further spectacular therapeutic progress, such as the availability of coagulation factors with a longer plasma half-life that allow for wider intervals between treatment. Moreover, new therapeutic products based on new mechanisms other than the replacement of the deficient factor, have become available (emicizumab) or are at an advanced stage of development. This review celebrates the success story of hemophilia care, while also discussing current limitations, issues and as yet unmet needs. The prospects of cure by means of gene therapy are also outlined.

\section{Introduction}

Among the more than 6,000 human diseases caused by single gene defects, ${ }^{1}$ the plasma deficiencies of coagulation proteins are of great importance to the hematologist, entailing as they do a lifelong bleeding tendency with important morbidity and mortality if not adequately managed. Inherited coagulation deficiencies are rare diseases according to the definitions adopted in the United States (less than 200,000 cases nationwide) and Europe (less than 5 cases per 10,000 persons in the general population). ${ }^{2}$ The hemophilias are clinically relevant rare diseases: hemophilia A (HA), which results from the deficiency or dysfunction of coagulation factor VIII (FVIII), and hemophilia B (HB) of factor IX (FIX). Both are due to mutations in genes located on chromosome $\mathrm{X}$ and thus largely affect males, with bleeding symptoms roughly proportional to the degree of factor deficiency in plasma. The main sites of spontaneous bleeding are joints and muscles, which, if inadequately treated, cause chronic damage to the musculoskeletal system resulting in severe handicaps and disability. Furthermore, trauma and surgical interventions are accompanied by uncontrolled bleeding.

A recent report on the worldwide distribution ${ }^{3}$ shows that the hemophilias are more frequent than previously estimated: 17.1 cases per 100,000 males with HA for all degrees of FVIII deficiency, 3.8 cases per 100,000 of HB, with a prevalence of 6 per 100,000 for HA and 1.1 per 100,000 for HB of cases with complete plasma factor deficiency, and thus a more severe clinical phenotype (Table 1). ${ }^{3}$ Inherited coagulation disorders are much rarer. These are due to defects in genes encoding other factors, such as fibrinogen, prothrombin, factors V, VII, X, XI and XIII. ${ }^{4}$ The defective genes are transmitted with an autosomal recessive pattern of inheritance and thus affect both sexes at similar rates. Prevalence rates in the general population range between 1 case per 500,000 for the more frequent factor VII deficiency and 1 in 2-3 million for the rarest prothrombin and factor XIII deficiencies (Table 1). ${ }^{4}$

The natural history and clinical phenotype of rarer coagulopathies are less accu-
Ferrata Storti Foundation
Haematologica 2020
Volume 105(3):545-553

\section{Correspondence:}

PIER MANNUCCIO MANNUCCI

piermannuccio.mannucci@policlinico.mi.it

Received: December 4, 2019.

Accepted: January 20, 2020.

Pre-published: February 14, 2020.

doi:10.3324/haematol.2019.232132

Check the online version for the most updated information on this article, online supplements, and information on authorship \& disclosures: www.haematologica.org/content/105/3/545

(C)2020 Ferrata Storti Foundation

Material published in Haematologica is covered by copyright. All rights are reserved to the Ferrata Storti Foundation. Use of published material is allowed under the following terms and conditions:

https://creativecommons.org/licenses/by-nc/4.0/legalcode. Copies of published material are allowed for personal or internal use. Sharing published material for non-commercial purposes is subject to the following conditions:

https://creativecommons.org/licenses/by-nc/4.0/legalcode, sect. 3. Reproducing and sharing published material for commercial purposes is not allowed without permission in writing from the publisher. 
rately established than for the hemophilias, but, in general, they tend to be less clinically severe at the same level of plasma deficiency. ${ }^{4,5}$ The inherited bleeding disorder von Willebrand disease (vWD) is not included among coagulation disorders because the primary defect is in the gene encoding the huge multimeric protein von Willebrand factor (vWF), essential for platelet-vessel wall interactions and the formation of the primary hemostatic plug. ${ }^{6}$ However, in vWD, there is often the additional deficiency of coagulation FVIII secondary to the primary defect of vWF that functions as a physiological stabilizer of FVIII to which is complexed in blood, and thus explains mechanistically the secondary coagulation defect. ${ }^{6} \mathrm{vWF}$ is encoded by a large gene on chromosome 12 (band 12p13.31), and vWD is transmitted as an autosomal dominant trait or as a recessive trait in the most severe and rarest type 3 (prevalence: 1 in 1-2 million). ${ }^{6}$ The prevalence in the general population of clinically relevant cases is similar to that of $\mathrm{HA}^{7}$ although mild vWF deficiencies of little clinical significance are much more frequent in the frame of population studies. ${ }^{8}$ In general, most patients with vWD are less severely affected clinically than those with the hemophilias, but they suffer more frequently from bleeding from mucosal tracts, such as epistaxis, menorrhagia, and gastrointestinal bleeding. ${ }^{6}$ Soft tissue bleeding, such as hemarthrosis and postoperative hemorrhages, is only frequent in cases associated with moderately severe FVIII deficiency, i.e. type 3 vWD. ${ }^{6}$

Besides this general background on the inherited coagulation disorders, in this article it will be emphasized that, in the last decade, there has been tremendous progress in the available therapeutic armentarium, particularly for patients with the hemophilias. Recent review articles show the progress regarding rare coagulation disorders and vWD.,9

\section{Early therapeutic progress in hemophilia}

One hundred years ago, at the time when Haematologica was first published, there was practically no treatment for the hemophilias or for the other inherited coagulation disorders. Whole blood was the only treatment approach available and this was of poor clinical efficacy (Figure 1), such that the life expectancy of hemophiliacs was 10-15 years, even in the most favorable circumstances. The few cases that survived were compromised by severe musculoskeletal damage that confined them to bed or to a wheelchair, and ice, analgesics and splinting were the only measures that could be used to alleviate

Table 1. Prevalence of inherited deficiencies of coagulation proteins and corresponding encoding genes and chromosomes.

\begin{tabular}{lcc}
$\begin{array}{l}\text { Protein } \\
\text { Fibrinogen }\end{array}$ & $\begin{array}{c}\text { Case prevalence in } \\
\text { the general population* }\end{array}$ & $\begin{array}{c}\text { Gene and } \\
\text { chromosome }\end{array}$ \\
Prothrombin & 1 in 1 million & $\begin{array}{c}F G, F G B(4 \mathrm{q} 31.3), \\
F G G(4 \mathrm{q} 32.1)\end{array}$ \\
\hline Factor V & 1 in 2 million & $F 2(11 \mathrm{p} 11.2)$ \\
Factor VII & 1 in 1 million & $F 5(1 \mathrm{q} 24.2)$ \\
Factor VIII & 1 in 500,000 & $F 7(13 \mathrm{q} 34)$ \\
Factor IX & 6 in 100,000 males & $F 8(\mathrm{x} 928)$ \\
\hline Factor X & 1 in 100,000 males & $F 9(\mathrm{x} 927.1)$ \\
Factor XI & 1 in million & $F 10(13 \mathrm{q} 34)$ \\
\hline Factor XIII & 1 in 1 million & $F 11(4 \mathrm{q} 35.2)$ \\
\hline
\end{tabular}

*Prevalences refer to the severe forms of the diseases due to homozygous or compound heterozygous gene mutations.

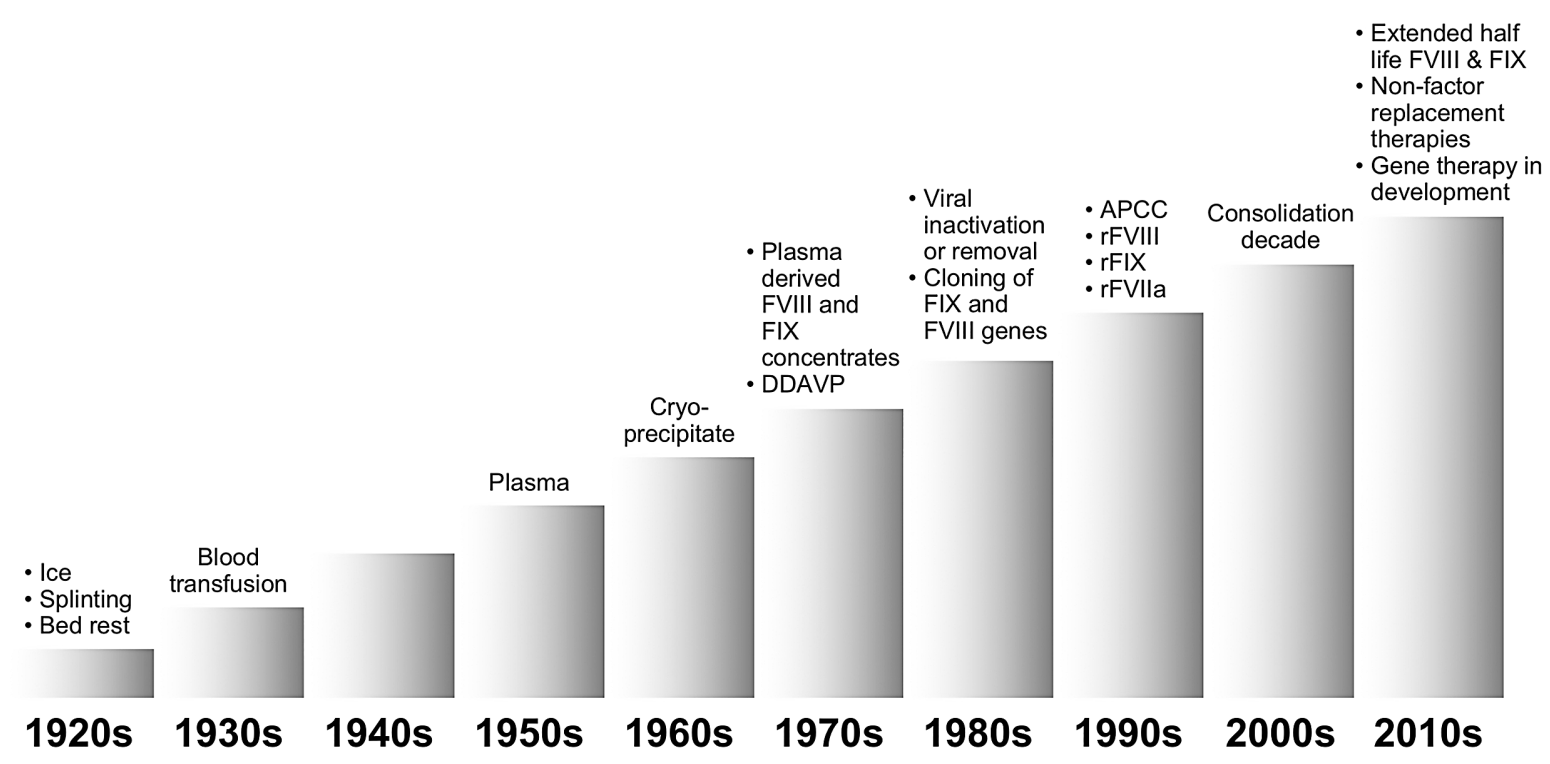

rFVIII = recombinant FVIII; rFIX = recombinant FIX; rFVIIa = activated recombinant FVII;

APCC $=$ activated prothrombin complex concentrates.

Figure 1. Progress in hemophilia therapy. Each decade of the last and current century features the main weapons available at the time for the treatment of patients with hemophilia. Each column represents a decade. 
pain and other symptoms associated with joint and muscle bleeding (Figure 1). The second World War and related combat casualties were triggers for the improved preparation of plasma, that contains all the coagulation factors (Figure 1). However, this form of replacement therapy was not widely available and of limited clinical efficacy. So, even until the 1960s, the life expectancy of patients with hemophilia was no more than 20-30 years.

A first step forward was the demonstration in 1964 by Judith Pool that cryoprecipitation of fresh-frozen plasma was able to concentrate FVIII (and also vWF and fibrinogen) in the pellet (Figure 1). But the most significant advance was seen in the 1970s with the industrial manufacturing and commercial availability of freeze-dried plasma concentrates of FVIII for HA and of the coagulation factors (II, VII, IX, X) of the so called prothrombin complex (PCC) for $\mathrm{HB}$ and the corresponding rare coagulopathies (Figure 1). The main advantages of these products was storage in simple refrigerators, reconstitution in small amounts of fluid, and no need for a drip to administrate blood, plasma and cryoprecipitate. Their availability, at least in European and North American countries and Japan, was the success story of the 1970s because they allowed home care and self-treatment. Some countries, such as Sweden, were also pioneers in using these products to develop prophylactic treatment of hemorrhages instead of only treating episodic bleeding events. ${ }^{10}$ Our demonstration in 1977 that the synthetic drug desmopressin (DDVP) was clinically efficacious as a non-transfusional form of FVIII replacement in mild HA and vWD contributed to further progress in the field. ${ }^{10}$

However, the 1980s threw a dramatic shadow on this favorable scenario when a large proportion of patients treated with factor produced from very large plasma pools developed serious or fatal blood-borne viral infections such as hepatitis and HIV/AIDS. ${ }^{11}$ Fortunately, this gloomy decade was accompanied by rapid progress in molecular medicine that not only clarified the genetic basis of the coagulation defects but also, and most importantly, led to the therapeutic production in the 1990s of recombinant coagulation FVIII and IX (Figure 1). Moreover, the addition of virucidal or virus-removal steps to the manufacturing process made plasma-derived coagulation products safer, such that no bloodborne viral infections have been reported since the late 1980s - early 1990s. ${ }^{11}$ The wider availability of safer and more effective therapies for hemophilia care attracted more attention among researchers and resulted in progress in what had so far been the rather hopeless management of a dire complication of HA: the development in at least one-third of patients of alloantibodies that make them refractory to replacement therapy, because the coagulant activity contained in FVIII replacement products is neutralized by specific inhibitors (and more rarely for FIX). ${ }^{11,12}$ In the late 1990 s, plasma concentrates of activated factors of the prothrombin complex (APCC), as well as the production of activated factor VII (rFVIIa) by recombinant DNA technology, offered novel ways to bypass the coagulation defect associated with FVIII inhibitors, and thus to improve the management of acute bleeding and surgical interventions (Figure 1). ${ }^{11,13}$ It was also demonstrated that inhibitory alloantibodies could be eradicated in approximately two-thirds of cases through the induction of immune tolerance (ITI) by means of the long-lasting and highly expensive administration of large doses of plasma-derived or recombinant FVIII prod- ucts, so that successful patients could resume replacement therapy and prophylaxis with efficacious outcomes. ${ }^{14,15} \mathrm{All}$ this progress improved not only the pattern and quality of patients' lives, but also led to substantial changes in their life expectancy, achieving figures very close to those of males without hemophilia in the general population; ${ }^{16-18}$ the figures are particularly encouraging if the ravages of the early years of uncontrolled HIV infection and AIDS are excluded..$^{3,17}$ The late 1990s and the whole first decade of the third millennium were years of consolidation and relatively slow progress (Figure 1), mainly characterized by the refinement of recombinant factors. There was a continuous improvement in the purity of these products, and the use of animal and human proteins during manufacturing and in the final formulation was avoided.

With this optimistic scenario of hemophilia care and of patients' life-expectancy, particularly in comparison with other monogenic diseases such as cystic fibrosis, thalassemia and muscular dystrophy, in the first decade of the new millennium efforts were mainly addressed to the formidable and still unresolved challenges of the global availability and affordability of replacement therapy and to the more widespread implementation of prophylaxis. ${ }^{19-21} \mathrm{On}$ the other hand, relatively little effort has been made to develop new therapeutic products. In contrast, multiple new therapies designed to address the challenges and the gaps in the standard treatments are now being developed.

\section{Recent progress in hemophilia therapy}

\section{Prophylaxis as standard of care}

Primary prophylaxis of bleeding episodes became the evidence-based standard of care following the randomized clinical trial of Manco-Johnson et al., ${ }^{22}$ who demonstrated that this preventive regimen was clearly superior to the episodic management of bleeds, because it reduced the rate of their occurrence and also achieved a marked reduction in joint damage. A subsequent randomized study by Gringeri et al. ${ }^{23}$ confirmed and strengthened this evidence, so that prophylaxis became the undisputed standard of care in countries that could afford it. Additional and important advantages were a much improved patient quality of life, including less hospitalizations and days lost from school and work, and an improved social life. However, the implementation of prophylaxis met some obstacles, ${ }^{20,21}$ in addition to that of affordability. ${ }^{24}$ The degree of adherence was often less than optimal, particularly in children and adolescents, owing to the burden created by the need of 2-3 or more weekly intravenous injections. This not only interfered with the patients' quality of life, but also created problems of vein access, with the related frequent need to resort to ports or other central venous access devices. ${ }^{20,21}$

\section{Extended plasma half-life coagulation factors}

Frequent intravenous injections are necessary due to the relatively short plasma half-life of replaced coagulation factors (range 10-14 hours for FVIII, 18-22 hours for FIX). Thus, starting from the 2010s, attempts were made to engineer these factors by recombinant technology, with the goal of obtaining medications that remained in the circulation longer and thus reducing the number of intravenous injections. Two main techniques were introduced: (i) coagulation factor fusion to proteins like the Fc part of 
IgG1 or albumin;,25,26 and (ii) conjugation with chemicals such as polyethylene glycol (PEG). ${ }^{27,28}$ The mechanism whereby albumin and Fc fusion prolongs the plasma halflife of coagulation factors is through the neonatal Fc receptor, ${ }^{25,29}$ which recycles them in plasma and thereby prolongs their effective circulation. ${ }^{29} \mathrm{PEG}$, attached randomly or site-specifically to coagulation factors, acts by slowing their degradation and renal elimination. ${ }^{27,28}$

Two extended half-life (EHL) recombinant coagulation factors were licensed in 2014: the Fc-fused FIX eftrenonacog alfa and the Fc-fused FVIII efmoroctocog alfa. $^{30,31}$ Subsequently, other EHL FVIII and FIX products were clinically evaluated, licensed and marketed (Tables 2 and 3), so that three pegylated FVIII products plus an albumin fusion and a pegylated FIX product, in addition to the two Fc- fusion products, are now available. ${ }^{32-40}$ FIX products can prolong the plasma half-life by from 4- to 5-fold (Table 3), whereas the half-life of FVIII can still be prolonged by no more than 1.5-1.7 fold (Table 2) due to its dependence on the half-life of its chaperone vWF to which it is complexed in blood. Pivotal clinical studies, conducted in highly selected adults and children, showed that these products were efficacious in stopping or preventing bleeding in the frame of episodic and prophylactic treatment regimens, and that they could also be used to safely manage surgical interventions. ${ }^{32-40}$

The median annualized bleeding rate (ABR), the parameter most commonly used to evaluate the clinical efficacy of antihemophilic products, ranged from 1 to 4 episodes, accumulating all the different prophylactic dosing regimens evaluated clinically for FVIII products. ${ }^{41}$ These ABR values compare favorably with those much higher values obtained with episodic regimens, ranging from 18 to $41 .^{41}$ For EHL FIX products, the ABR had a similar range of values with different prophylaxis regimens. ${ }^{31,40}$ In practice, EHL FVIII products can be effectively administered twice instead of thrice weekly, but most patients are not satisfactorily protected from bleeds with weekly dosing regimens. ${ }^{41}$ EHL FIX products are much more satisfactory, because they can be given every 10 or even 15 days,${ }^{42}$ and thus allow a lower annual burden of intravenous injections, the average reduction being more prominent $(\sim 60 \%)$ than for FVIII products $(-30 \%)$ (Figure 2$)$. Furthermore, higher trough levels of both FVIII (2-3\%) and FIX $(5-10 \%)$ could be achieved than with SHL products ${ }^{41,42}$ and there was, in general, a lower annual consumption in units of EHL products. ${ }^{41,42}$ From a practical clinical standpoint these considerations broadly apply to all the different products, and these may be considered equivalent in terms of efficacy at a time when no face-toface comparative clinical study is available. The market price of all these products is usually higher, but there are exceptions in countries where the price per unit of EHL FVIII is very close to that of SHL FVIII.

A few limitations warrant our attention. Despite higher trough plasma factor levels, the ideal goal of avoiding all

Table 2. Extended half-life factor VIII products.

\begin{tabular}{lcccc} 
Engineered protein & Year of first licensing & Manufacturer & Plasma half-life (hours) & Half-Ifie prolongation* \\
Efmoroctocog alfa & 2014 & Biogen/Sobi & 19 & $1.5-1.7$ \\
Rurioctocog alfa pegol & 2015 & Baxalta/Takeda & 14.3 & $1.3-1.5$ \\
\hline Danoctocog alfa pegol & 2018 & Bayer & 19 & 1.6 \\
Turoctocog alfa pegol & 2019 & Novo Nordisk & 18.4 & 1.6
\end{tabular}

${ }^{*}$ Calculated from an average plasma half-life of standard coagulation FVIII of approximately 12 hours.

Table 3. Extended half-life factor IX products.

\begin{tabular}{lcccc} 
Engineered protein & Year of first licensing & Manufacturer & Plasma half-Ifie (hours) & Half-life prolongation* \\
Efrenonacog alfa & 2014 & Biogen/Sobi & 4.3 & 101 \\
Albutrepenonacog alfa & 2016 & CSL Behring & 5.3 \\
\hline Nonacog beta pegol & 2017 & Novo Nordisk & 93 \\
\hline
\end{tabular}

*Calculated from an average plasma half-life of standard FIX products of approximately 19 hours.

\begin{tabular}{c} 
FVIII products \\
\hline Reduction of infusion number: $30 \%$ \\
Trough levels: $2-3 \mathrm{IU} / \mathbf{d L}$ \\
Patients with severe hemophilia $A$ are converted to a moderate phenotype
\end{tabular}

Half-life: 1.3-1.7 fold increase

\begin{tabular}{|c|}
\hline FIX products \\
\hline Reduction of infusion number: $\mathbf{6 0 \%}$ \\
Trough levels: $\mathbf{5 - 1 0}$ IU/dL \\
Patients with severe hemophilia B are converted to a mild phenotype \\
Half-life: $\mathbf{4 - 6}$ fold increase \\
\hline
\end{tabular}

Figure 2. Summary features of Factor VIII and Factor IX products with an extended half-life. Comparative main characteristics of the extended half-life coagulation factor products (left FVIII, right FX), including the percentage reduction of the annual infusion number compared with the standard half-life products, trough plasma factor levels that can be achieved, expected changes in the clinical phenotype the range of increase of plasma half-life. 
spontaneous bleeds was achieved in no more than 30$40 \%$ of cases in the prophylaxis dosing regimens evaluated in the pivotal studies, even though higher rates of zero bleeding were often obtained in the extension studies through the personalization of the dosing regimens. ${ }^{41}$ Because these products have only been marketed for six years or less, no evaluation of long-term, real-life experience has yet been made. No theoretical concerns regarding a higher rate of inhibitor development with these highly engineered coagulation factors were raised in the pivotal studies, ${ }^{41}$ although only previously-treated patients at low risk of developing this complication were enrolled. It remains to be seen whether or not this risk is smaller, equal or higher than that of the SHL recombinant or plasmatic products in high-risk patients, i.e. those not previously treated with any source of FVIII and thus with no tolerance of this moiety. Finally, four of the seven currently marketed EHL products use an exogenous chemical such as PEG. Pegylation has been safely used to prolong the length of time medication remains in the circulation in several products, e.g. epoietin, interferon, the human growth factor, and many others. ${ }^{28}$ The amount of PEG used to prolong the half-life of FVIII, present in tiny amounts in plasma, is the smallest among all the pegylated medications, but, as yet, this does not apply for FIX ${ }^{43}$ On the other hand, none of the currently licensed pegylated medications is administered lifelong from birth, a unique situation for antihemophilic factors. For the moment, in contrast to the US Food and Drug Administration (FDA), the European Medicines Agency (EMA) has chosen to restrict the use of most pegylated coagulation factors to patients over the age of 12 years.

\section{Non-factor therapies}

In spite of the progress made with the availability of EHL factors, unmet needs remained. In HA patients without inhibitors, the reduction in the frequency of intravenous injections was not considered satisfactory, ${ }^{41,42}$ and therapy still based on the need for a venous access continued to be unattractive. HA patients with FVIII inhibitors remained poor candidates for prophylaxis that could only be provided by bypassing products such as APCC and rFVIIa that are very expensive ${ }^{44}$ and difficult to administer on a regular preventive basis. With these drawbacks in mind, therapeutic approaches that were not based on the replacement of the deficient factor were developed. This took place in two main ways: (i) for HA, by mimicking the coagulant activity of FVIII; and (ii) for both HA and HB, by increasing defective thrombin formation through the inhi- bition of the naturally occurring anticoagulants (antithrombin, tissue factor pathway inhibitor, and activated protein $\mathrm{C}$ ). For the moment, only the monoclonal antibody emicizumab that mimics FVIII activity has been licensed and marketed. The approach of quenching the anticoagulant pathways, potentially applicable not only to both the hemophilias but also to all the inherited coagulation disorders, is currently undergoing an advanced stage of clinical development, but since no product has been licensed yet, here this will receive less attention than emicizumab.

\section{Emicizumab}

This bispecific monoclonal antibody supports the spatial interaction between activated FIX (FIXa) and factor X, and thereby promotes thrombin formation by mimicking FVIIIa activity regardless of FVIII deficiency and the presence of FVIII inhibitors. ${ }^{45}$ Administered subcutaneously, this drug reaches a steady state with a long plasma halflife that allows well-spaced dosing intervals of at least every week or even every two weeks. ${ }^{46,47}$ It was first licensed in 2017 in the USA, and then in Europe and Japan, for the prophylaxis of bleeding in adults and pediatric patients with HA, with and without inhibitors. The first two pivotal studies were carried out in patients with FVIII inhibitors: HAVEN 1 in adults and adolescents, ${ }^{48}$ and HAVEN 2 in pediatric patients under 12 years of age. ${ }^{49}$ Using various dosing regimens and intervals between the subcutaneous injections, both the studies found that the $\mathrm{ABR}$ ranged between 0.2 and 2.9 , values that compare favorably with those observed in inhibitor patients not on prophylaxis (Table 4). The rate of zero bleeding events ranged between $63 \%$ and $90 \%$, the highest rate being obtained in HAVEN 2 when the medication was given at a dosage of $3 \mathrm{mg} / \mathrm{Kg}$ of body weight every two weeks (Table 4). These results are impressive if one considers that, until now, HA patients with FVIII inhibitors have been poor candidates for a feasible prophylaxis of bleeding, because attempts to use aPCC or rFVIII were jeopardized by the need for very frequent intravenous injections, let alone the very high costs. ${ }^{44}$ Following the striking results obtained in HAVEN 1 and 2, emicizumab has also been evaluated in HA patients without inhibitors. ${ }^{50,51}$ In the HAVEN 3 study, previously treated adult patients were assigned to subcutaneous emicizumab administered weekly or even every two weeks; study participants had much lower ABR than those not on prophylaxis (1.5 and 1.3 vs. 38.2) and much higher rates of zero bleeds (50\% and $40 \%$ vs. $0 \%$ ) (Table 4 ).

Table 4. Bleeding rates observed with different emicizumab dosing regimen(s) in patients with hemophilia A with and without inhibitors in the context of the HAVEN studies.

\begin{tabular}{lccc} 
STUDY & DOSING REGIMEN & ABR (MEDIAN) & ZERO BLEEDING RAIES \\
HAVEN 1 & $1 \times W$ prophylaxis $(1.5 \mathrm{mg} / \mathrm{kg})(\mathrm{n}=35)$ & 2.9 & $63 \%$ \\
& No prophylaxis $(\mathrm{n}=18)$ & 23.3 & $6 \%$ \\
HAVEN 2 & $1 \times W$ prophylaxis $(1.5 \mathrm{mg} / \mathrm{kg})(\mathrm{n}=68)$ & 0.3 & $76.9 \%$ \\
& E2W prophylaxis $(3.0 \mathrm{mg} / \mathrm{kg})(\mathrm{n}=10)$ & 0.2 & $90 \%$ \\
& E4W prophylaxis $(6 \mathrm{mg} / \mathrm{kg})(\mathrm{n}=10)$ & 2.2 & $60 \%$ \\
\hline HAVEN 3 & $1 \times W$ prophylaxis $(1.5 \mathrm{mg} / \mathrm{kg})(\mathrm{n}=36)$ & 1.5 & $50 \%$ \\
& E2W prophylaxis $(3.0 \mathrm{mg} / \mathrm{kg})(\mathrm{n}=35)$ & 1.3 & $40 \%$ \\
HAVEN 4 & No prophylaxis $(\mathrm{n}=18)$ & 38.2 & 0 \\
\hline
\end{tabular}

ABR: annualized bleeding rate; NR: not reported; $1 \times W$ : once weekly; E2W: every 2 weeks; E4W: every 4 weeks. ${ }^{a}$ Median ABR during the expansion phase. 
All in all, the main benefit of this first non-factor replacement antihemophilic medication is the feasibility of regular prophylaxis in patients with inhibitors, using the advantageous and user-friendly subcutaneous administration route at weekly intervals or even less frequently. The high cost of emicizumab is an issue, but all the products used so far to manage patients with inhibitors are very expensive, including the traditional bypassing agents and immune tolerance inductions (ITI). Furthermore, the licensing of emicizumab also for HA patients without inhibitors is an important alternative to the currently available options of SHL and EHL coagulation factors, with the advantage of the subcutaneous instead of the intravenous route of administration. Efficacy parameters, such as the ABR and the zero bleeding rates, appear to be better than those obtained with the EHL FVIII products, but since there have still not been any face-to-face comparison studies between replacement and non-replacement products, this impression stems from indirect data.

A potential but still unexplored approach is the use of this non-FVIII product in young, previously untreated patients (PUP) with severe HA who, at high risk of developing inhibitors following exposure to FVIII replacement, might be able to avoid this complication. Another definite advantage is the subcutaneous route of administration, which would promote early prophylaxis without the need for venous ports. Emicizumab may also help to prevent the intracranial hemorrhages that are relatively frequent in the early years of life. Potential disadvantages may materialize in cases in which FVIII replacement is required to prevent or treat breakthrough bleeds, because the delayed FVIII inhibitors may develop in dangerous, high-risk circumstances, such as at the time of major trauma or surgery. Thus, the use of emicizumab in PUP is still a subject of debate and warrants a specific study to evaluate the forementioned advantages and disadvantages of this approach.

Other unanswered questions and causes for concern remain (Table 4). It is still not known whether or not the FVIII-mimicking activity of emicizumab provides the same physiological benefits of the bona fide coagulation factor, such as the long-term preservation of joint and bone health and the optimal support of wound healing. ${ }^{52}$ In pivotal studies, a few patients developed thrombotic microangiopathies and other thromboses when inhibitor patients had a bleeding episode and were concomitantly treated with large and frequent doses of APCC. ${ }^{48}$ At least 23 deaths have been associated with the use of emicizum$\mathrm{ab}$, mostly in patients with inhibitors, but also in some without. The deaths occurred both in the context of the pivotal clinical studies and as a result of the expanded access, compassionate and post marketing use, as reported by the manufacturer ${ }^{53}$ and by the FDA Adverse Events Reporting System (FEARS). ${ }^{54}$ As emphasized by Aledort, ${ }^{55}$ more information on causality or chance association is needed to dissipate the uncertainty that surrounds these cases among consumers and care-givers.

\section{Other non-factor therapies}

Medications with mechanisms of actions other than that of emicizumab, and also mainly administered subcutaneously, are currently at an advanced stage of clinical development. Concizumab, a monoclonal antibody against the anticoagulant protein TFPI, increases the potential for thrombin generation. ${ }^{56,57} \mathrm{~A}$ trend towards lower bleeding rates was observed in patients with $\mathrm{HA}$ and $\mathrm{HB}$ with and without inhibitors, but the cases were too few to provide robust evidence of efficacy. Another anti-TFPI monoclonal antibody is PF-06741086, currently undergoing a phase II trial (NCT02974855)..$^{58}$ Fiturisan is a compound that interferes with RNA, and that decreases the plasma concentrations of antithrombin. ${ }^{59,60}$ In early clinical studies, this agent, given subcutaneously at progressively higher dosages and even at monthly intervals, was accompanied by the progressive decrease in plasma antithrombin paralleled by an increase in thrombin generation and reduction of the ABR. Phase III studies of fiturisan in patients with severe $\mathrm{HA}$ and $\mathrm{HB}$ with and without inhibitors are at the advanced phase of development, ${ }^{61,62}$ but the drug is as yet not licensed for clinical use. A fatal thrombotic event that occurred in 2017 in a patient with severe HA during a phase II study ${ }^{63}$ led to the FDA temporarily stopping the study, but some protocol and guideline changes have allowed it to be restarted and to move this product forward to phase III studies; these are currently ongoing. On the whole, it is still too early to truly understand the role of these additional non-factor products in the scenario of hemophilia care, but potential advantages are their use not only in HA but also in HB and other inherited coagulation disorders, with or without inhibitors. Another advantage is that they can be administered at intervals that are as widely spaced as once a month. ${ }^{59,60}$

\section{Gene therapy}

As we have seen, within the already positive scenario that developed at the beginning of the third millennium in terms of almost normal life expectancy, a new era of huge innovation in hemophilia therapy is currently underway. ${ }^{64}$ A recent study attempted to identify the main players involved in this almost miraculous progress. These include outstanding and dedicated physician-scientists, patient advocates and consumer organizations, but also more and more pharmaceutical companies involved in the hemophilia market. ${ }^{64}$ With this background, are there still reasons and incentives for further progress? The main thrust stems from patients, who want to be cured! For them, cure means to be free of spontaneous bleeds, because the ideal zero rate has not been fully achieved with the available weaponry. ${ }^{65}$ Thus, the ideal goal is gene therapy, preferably with a single therapeutic intervention of lifelong duration.

The first vector associated with curative gene transfer in animal models of hemophilia was the adeno-associated virus (AAV), and, so far, AAV vectors are the only tools used to achieve therapeutic levels of FVIII and IX in hemophilia patients. ${ }^{66-70}$ Historically, the first study involved ten patients with severe $\mathrm{HB}$ at the Royal Free Hospital in London, UK, who received single but increasing doses of an AAV8 vector, some of them with a current follow up of 9-10 years. ${ }^{71,72}$ They continue to have stable expression of the transgene, with plasma levels ranging from $2 \%$ to $5 \%$, and a $90 \%$ reduction in bleeding episodes. ${ }^{72}$ Among the six additional ongoing studies in $\mathrm{HB}^{73}$ impressive results were obtained in the SPK-9001 phase I-II study, of particular interest because it used an AAV8 vector expressing the gain-of-function FIX Padua gene mutation. ${ }^{74}$ The 15 patients who received a single infusion of this vector attained mean plasma levels of FIX of $33.7 \%$ (range 14.3$76.8 \%$ ) over a period of at least 52 weeks. ${ }^{74}$ According to 
the manufacturers, in the SPK-9001 study, a single vector infusion was accompanied by a $98 \%$ reduction in the $\mathrm{ABR} ;{ }^{75}$ this has led to a pivotal phase II study, which is currently undergoing. ${ }^{76}$ Satisfactory plasma FIX levels were also obtained in the phase I-II studies BAX335 and AMT060, using an AAV8 vector. ${ }^{7678}$ In both studies, FIX levels remained stable for 2-3 years and ABR were reduced, such that a phase III AMT-061 study has begun. On the whole, at least $56 \mathrm{HB}$ patients have been treated with various serotypes of AAV vectors (mainly AAV5 and AAV8), and long-term expression of the transgene was obtained after a single intravenous infusion of the vector. ${ }^{73}$

The use of gene transfer was initially slower in HA, due to the much larger size of the FVIII gene that made it difficult to pack the corresponding cDNA in AAV vectors. This problem was tackled by using a B-domain delated human FVIII cDNA endowed with a liver-specific promoter. ${ }^{79,80}$ Striking clinical results in HA were first published in 2017 using increasing dosages of the vector AAV5-hFVIII-SO (valoctogene roxaparvovec). ${ }^{81}$ One year after a single administration, median FVIII in plasma was $77 \%$ and ranged from 19 to as high as $164 \% .{ }^{81}$ Importantly, the mean ABR decreased by $97 \%$ from previous values. According to a recent update of the results obtained in 13 patients at year 2 after gene transfer, ${ }^{82}$ two of them had normal plasma FVIII levels $(52 \%$ and $86 \%)$, ten had values within the range of mild HA (from $6 \%$ to $38 \%$ ), and one had levels (4\%) compatible with moderate HA. All these patients had experienced a dramatic reduction in the incidence of bleeds, in spite of the fact that they had stopped FVIII prophylaxis. These excellent results were substantially maintained in the eight patients who were also evaluated at year 3 after gene transfer. ${ }^{82}$ Additional patients are currently being recruited into a phase III trial, which has set three ambitious goals: (i) enrollment of 130 patients; (ii) to obtain stable FVIII levels of at least $40 \%$; and (iii) to demonstrate superiority over the traditional therapies. At least five additional trials of gene therapy for HA are currently ongoing using various AAV serotypes as vectors. The results available so far confirm the efficiency and durability of AAVmediated gene therapy. However, at the moment, maximum follow up is no longer than 1.5 years, ${ }^{73}$ and, as for all new therapeutic developments, long-term follow up is still required to firmly consolidate the safety profile.

There are still important problems and issues that need to be resolved before licensing procedures and availability of gene therapy for the hemophilias can be carried forward. Only adult patients have been enrolled in studies so far, because in pediatric patients, the active dividing hepatocytes of children mean that there is no guarantee of achieving a persistent expression of a non-integrating vector, such as AAV. On the other hand, the long-term expression that followed a single vector infusion in the first UK-based FIXdeficient patients is surprising, because, according to the physiological turnover rate of hepatocytes $10 \%$ per year in adults), a transgene expression drop by $50 \%$ should have occurred within the first five years of treatment. Thus, some degree of integration of the transgene has perhaps taken place in the host DNA, with a theoretical risk of genotoxicity and cancer development. ${ }^{83,84}$ Only liver biopsies can truly establish the fate of the AAV genome, and to what extent it is episomal or DNA integrated. ${ }^{85.86}$ Liver biopsies would also be useful to establish the histological consequences of the most significant adverse effect observed so far in practically all the gene transfer studies, that is, a dosedependent increase in transaminases that was controlled by means of short periods of corticosteroid administration. ${ }^{73,87}$ It remains to be established whether or not these flares of hepatocyte cell necrosis, called inappropriately "transaminitis", are causing chronic liver damage. Normally in the context of AAV only a small percentage of the hepatocyes are stably transduced and responsible for long-term expression (in a serotype and dose-dependent manner). However, liver biopsies are not necessary informative on the either acute (transient transaminitis) or histological changes if the results are "normal" or negative. Moreover, follow up beyond 2-3 years is still awaited for most studies, so it is not clear how long transgene expression is going to be maintained. This is important, because immune reactions to the AAV capsid lead to the formation of neutralizing antibodies that prevent effective repeat of vector delivery, at least with the same AAV serotype. ${ }^{7,87}$ Moreover, there are still no models of payment for gene therapy in hemophilia. BioMarin, the California-based pharmaceutical company that sponsored the forementioned study of gene transfer in $\mathrm{HA}^{81,1,82}$ recently declared to the Wall Street Journal that, once licensed, they are planning to price their product (brand name: Valrox) between 2 and 3 million dollars. This would make it the most expensive medication in the world, even more expensive than the current 'leader' Zolgensma, priced at 2.1 million dollars for patients with spinal muscular dystrophy.

Once these challenges to gene therapy in the hemophilias are solved, for a number of reasons, this is likely to become the treatment of choice. Despite the major advances in prophylaxis obtained with EHL factor products and non-factor therapies, breakthrough bleeding has still not been fully eliminated, and treatment is still invasive, both physically and psychologically, even when the subcutaneous route of administration is used. Patients with severe hemophilia live with the risk of bleeding every day of their lives, and no repeated dosing regimen will be able to replace the advantages of a one-off lifetime cure. Finally, we should not forget that $70 \%$ of patients worldwide have no treatment, either because nothing is available or because they cannot afford it; life expectancy at birth for these patients is still only ten years or less! The World Federation of Hemophilia has been attempting to tackle this formidable problem since its foundation in 1967, but despite great progress in medium-income countries, the great majority of low-income countries are still in the same situation they were in 100 years ago: ice, splinting, bed rest, and blood transfusions when available!

\section{References}

1. Jackson M, Marks L, May GHW, Wilson JB. The genetic basis of disease. Essays Biochem. 2018;62(5):643-723.

2. Khosla N, Valdez R. A compilation of national plans, policies and government actions for rare diseases in 23 countries. Intractable Rare Dis Res. 2018;7(4):213-222. 3. Iorio A, Stonebraker JS, Chambost H, et al. Establishing the prevalence and prevalence at birth of hemophilia in males: a meta-analytic approach using national registries. Ann Intern Med. 2019;171:540-546.
4. Palla R, Peyvandi F, Shapiro AD. Rare bleeding disorders: diagnosis and treatment. Blood. 2015;125(13):2052-2061.

5. Menegatti M, Peyvandi F. Treatment of rare factor deficiencies other than hemophilia. Blood. 2019;133(5):415-424.

6. Leebeek FW, Eikenboom JC. Von 
Willebrand's disease. N Engl J Med. 2016; 375(21):2067-2080

7. Sadler JE, Mannucci PM, Berntorp E. Impact, diagnosis and treatment of von Willebrand disease. Thromb Haemost. 2000;84(2):160174

8. Rodeghiero F, Castaman G, Dini E. Epidemiological investigation of the prevalence of von Willebrand's disease. Blood. 1987;69(2):454-459.

9. Mannucci PM. New therapies for von Willebrand disease. Blood Adv. 2019;3(21): 3481-3487.

10. Mannucci PM, Ruggeri ZM, Pareti FI, Capitanio A. 1-Deamino-8-d-arginine vasopressin: a new pharmacological approach to the management of haemophilia and von Willebrands' diseases. Lancet. 1977 23;1 (8017):869-872

11. Peyvandi F, Garagiola I, Young G. The past and future of haemophilia: diagnosis, treatments, and its complications. Lancet. 2016;388(10040):187-197

12. Wight J, Paisley S. The epidemiology of inhibitors in haemophilia A: a systematic review. Haemophilia. 2003;9(4):418-435.

13. Rocino A, Franchini M, Coppola A. Treatment and prevention of bleeds in haemophilia patients with inhibitors to factor VIII/IX. J Clin Med. 2017;6(4): E46.

14. DiMichele DM, Hoots WK, Pipe SW, Rivard GE, Santagostino E. International workshop on immune tolerance induction: consensus recommendations. Haemophilia. 2007;13 (Suppl 1):1-22

15. Hay CR, DiMichele DM; International Immune Tolerance Study. The principal results of the International Immune Tolerance Study: a randomized dose comparison. Blood. 2012;119(6):1335-1344

16. Plug I, Van Der Bom JG, Peters M, et al. Mortality and causes of death in patients with hemophilia, 1992-2001: a prospective cohort study. J Thromb Haemost. 2006;4(3): 510-516.

17. Darby SC, Kan SW, Spooner RJ, et al. Mortality rates, life expectancy, and causes of death in people with hemophilia A or B in the United Kingdom who were not infected with HIV. Blood. 2007;110(3):815-825.

18. Tagliaferri A, Rivolta GF, Iorio A, et al. Mortality and causes of death in Italian persons with haemophilia, 1990-2007. Haemophilia. 2010;16(3):437-446.

19. Carcao MD, Aledort L. Prophylactic factor replacement in hemophilia. Blood Rev. 2004;18(2):101-113.

20. Bolton-Maggs PH. Optimal haemophilia care versus the reality. Br J Haematol. 2006; 132(6):671-682

21. Saxena K. Barriers and perceived limitations to early treatment of hemophilia. J Blood Med. 2013;4:49-56.

22. Manco-Johnson MJ, Abshire TC, Shapiro $\mathrm{AD}$, et al. Prophylaxis versus episodic treatment to prevent joint disease in boys with severe hemophilia. N Engl J Med. 2007;357 (6):535-544.

23. Gringeri A, Lundin B, von Mackensen S, Mantovani L, Mannucci PM; ESPRIT Study Group. A randomized clinical trial of prophylaxis in children with hemophilia $\mathrm{A}$ (the ESPRIT Study). J Thromb Haemost. 2011;9 (4):700-710.

24. Chen SL. Economic costs of hemophilia and the impact of prophylactic treatment on patient management. Am J Manag Care. 2016;22(5 Suppl):s126-133.

25. Dumont JA, Liu T, Low SC, et al. Prolonged activity of a recombinant factor VIII-Fc fusion protein in hemophilia A mice and dogs. Blood. 2012;119(13):3024-3030.

26. Schulte S. Innovative coagulation factors: albumin fusion technology and recombinant single-chain factor VIII. Thromb Res 2013;131 (Suppl. 2):s2-s6

27. Ivens IA, Baumann A, McDonald TA, et al. PEGylated therapeutic proteins for haemophilia treatment: a review for haemophilia caregivers. Haemophilia. 2013;19(1):11-20.

28. Swierczewska M, Lee KC, Lee S. What is the future of PEGylated therapies? Expert Opin Emerg Drugs. 2015;20(4):531-536.

29. Roopenian DC, Akilesh S. FcRn: the neonatal Fc receptor comes of age. Nat Rev Immunol. 2007;7(9):715-725.

30. Powell JS, Josephson NC, Quon D, et al. Safety and prolonged activity of recombinant factor VIII Fc fusion protein in hemophilia A patients. Blood. 2012;119(13):30313037

31. Shapiro AD, Ragni MV, Valentino LA, et al Recombinant factor IX-Fc fusion protein ( $\mathrm{rFIXFc}$ ) demonstrates safety and prolonged activity in a phase $1 / 2$ a study in hemophilia B patients. Blood. 2012;119(3):666-672.

32. Mahlangu J, Powell JS, Ragni MV, et al. Phase 3 study of recombinant factor VIII Fc fusion protein in severe hemophilia A. Blood. 2014;123(3):317-325

33. Konkle BA, Stasyshyn O, Chowdary P, et al. Pegylated, full-length, recombinant factor VIII for prophylactic and on-demand treatment of severe hemophilia A. Blood. 2015;126(9):1078-1085

34. Mullins ES, Stasyshyn O, Alvarez-Román MT, et al. Extended half-life pegylated, fulllength recombinant factor VIII for prophylaxis in children with severe haemophilia A. Haemophilia. 2017;23(2):238-246.

35. Reding MT, Ng HJ, Poulsen LH, et al. Safety and efficacy of BAY 94-9027, a prolongedhalf-life factor VIII. J Thromb Haemost. 2017;15(3):411-419

36. Santagostino E, Lalezari S, Reding MT, et al. Safety and efficacy of BAY 94-9027, an extended-half-life factor VIII, during surgery in patients with severe hemophilia A: Results of the PROTECT VIII clinical trial. Thromb Res. 2019;183:13-19.

37. Giangrande P, Andreeva T, Chowdary P, et al. Clinical evaluation of glycoPEGylated recombinant FVIII: Efficacy and safety in severe haemophilia A. Thromb Haemost. 2017;117(2):252-261

38. Meunier S, Alamelu J, Ehrenforth S, et al. Safety and efficacy of a glycoPEGylated rFVIII (turoctocog alpha pegol, N8-GP) in paediatric patients with severe haemophilia A. Thromb Haemost. 2017;117(9):17051713

39. Gruppo R, López-Fernández MF, Wynn TT, Engl W, Sharkhawy M, Tangada S. Perioperative haemostasis with full-length, PEGylated, recombinant factor VIII with extended half-life (rurioctocog alfa pegol) in patients with haemophilia A: Final results of a multicentre, single-arm phase III trial. Haemophilia. 2019;25(5):773-781

40. Santagostino E, Negrier C, Klamroth R, et al. Safety and pharmacokinetics of a novel recombinant fusion protein linking coagulation factor IX with albumin (rIX-FP) in hemophilia B patients. Blood. 2012;120(12): 2405-2411.

41. Aledort L, Mannucci PM, Schramm W, Tarantino M. Factor VIII replacement is still the standard of care in haemophilia A. Blood Transfus 2019:17:479-486

42. Mancuso ME, Santagostino E. Outcome of clinical trials with new extended half-life
FVIII/IX concentrates. J Clin Med. 2017;6: E39.

43. Baumann A, Piel I, Hucke F, Sandmann S, Hetzel T, Schwarz T. Pharmacokinetics, excretion, distribution, and metabolism of 60-kDa polyethylene glycol used in BAY 949027 in rats and its value for human prediction. Eur J Pharm Sci. 2019 Mar 15;130:11-20

44. Gringeri A, Mantovani LG, Scalone L, Mannucci PM; COCIS Study Group. Cost of care and quality of life for patients with hemophilia complicated by inhibitors: the COCIS Study Group. Blood. 2003;102(7): 2358-2363.

45. Kitazawa T, Igawa T, Sampei Z, et al. A bispecific antibody to factors IXa and $X$ restores factor VIII hemostatic activity in a hemophilia A model. Nat Med. 2012;18(10): 1570-1574.

46. Uchida N, Sambe T, Yoneyama K, et al. A first-in-human phase 1 study of ACE910, a novel factor VIII-mimetic bispecific antibody, in healthy subjects. Blood. 2016;127(13):1633-1641

47. Shima $M$, Hanabusa $H$, Taki $M$, et al. Factor VIII-mimetic function of humanized bispecific antibody in hemophilia A. N Engl Med. 2016;374(21):2044-2053

48. Oldenburg J, Mahlangu JN, Kim B, et al Emicizumab prophylaxis in hemophilia A with inhibitors. N Engl J Med. 2017;377 (9):809-818

49. Young G, Liesner R, Sidonio R, et al Emicizumab Prophylaxis Provides Flexible and Effective Bleed Control in Children with Hemophilia with Inhibitors: Results from the HAVEN 2 Study. Paper presented at: American Society of Hematology Annual Meeting; December 14 4, 2018; San Diego

50. Mahlangu J, Oldenburg J, Paz-Priel I, et al. Emicizumab prophylaxis in patients who have hemophilia $\mathrm{A}$ without inhibitors. N Engl J Med. 2018;379(9):811-822.

51. Pipe SW, Shima M, Lehle M, et al. Efficacy, safety, and pharmacokinetics of emicizumab prophylaxis given every 4 weeks in people with haemophilia A (HAVEN 4): a multicentre, open-label, non-randomised phase 3 study. Lancet Haematol. 2019;6(6):e295e305.

52. Samuelson Bannow B, Recht M, Négrier C, et al. Factor VIII: long-established role in haemophilia $\mathrm{A}$ and emerging evidence beyond haemostasis. Blood Rev. 2019;35:4350

53. Fatalities in emicizumab-kxwh clinical trials, expanded access,compassionate use, and the postmarketing setting. South San Francisco, CA: Genentech, 2018 (https://www.emicizumabinfo.com/content/dam/gene/emicizumabinfo/hcp/pdfs/fa talities.pdf)

54. FDA adverse events reporting system (FAERS) public dashboard: FAERS cases 14455349 and 16262656. Rockville, MD: Food and Drug Administration (https://fis.fda. gov/sense/app/d10be6bb494e-4cd2-82e4-0135608ddc13/sheet/ 33a0f68e-45c-48e2-bc81-8141c6aaf772/ state/ analysis).

55. Aledort LM. Deaths associated with emicizumab in patients with hemophilia A. N Engl J Med. 2019:381(19):1878-1879.

56. Chowdary P, Lethagen S, Friedrich U, et al Safety and pharmacokinetics of anti-TFPI antibody (concizumab) in healthy volunteers and patients with hemophilia: a randomized first human dose trial. J Thromb Haemost. 2015;13(5):743-754

57. Eichler H, Angchaisuksiri P, Kavakli K, et al A randomized trial of safety, pharmacoki- 
netics and pharmacodynamics of concizum$\mathrm{ab}$ in people with hemophilia A. J Thromb Haemost. 2018;16(11):2184-2195.

58. Gu JM, Zhao XY, Schwarz T, et al. Mechanistic modeling of the pharmacodynamic and pharmacokinetic relationship of tissue factor pathway inhibitor-neutralizing antibody (BAY 1093884) in cynomolgus monkeys. AAPS J. 2017;19(4):1186-1195.

59. Pasi KJ, Rangarajan S, Georgiev P, et al. Targeting of antithrombin in hemophilia A or B with RNAi therapy. N Engl J Med. 2017;377(9):819-828.

60. Ragni MV, Georgiev P, Mant T, et al. Fitusiran, an investigational RNAi therapeutic targeting antithrombin for the treatment of hemophilia: updated results from a phase 1 and phase 1/2 extension study in patients without inhibitors [abstract]. Blood. 2016;128(22):2572

61. ClinicalTrials.gov. A Study study of fitusiran (ALN-AT3SC) in severe hemophilia A and B patients with inhibitors (ATLAS-INH). Identifier: NCT03417102. 2018. https://clinicaltrials.gov/ct2/show/NCT03417102. Accessed February, 2019.

62. Clinicaltrials.gov. A study of fitusiran (ALNAT3SC) in severe hemophilia A and B patients without inhibitors. Identifier: NCT03417245. 2018. https://clinicaltrials.gov/ct2/show/NCT034 17245. Accessed June, 2019.

63. Alnylam. Alnylam provides pipeline update on fitusiran and givosiran investigational RNAi therapeutic programs. 2017. http://investors.alnylam.com/news-releases/news-release-details/alnylam-providespipeline-update-fitusiran-and-givosiran. Accessed February, 2019.

64. Mannucci PM. Miracle of haemophilia drugs: Personal views about a few main players. Haemophilia. 2018;24:557-562.

65. von Mackensen S, Kalnins W, Krucker J, et al. Haemophilia patients' unmet needs and their expectations of the new extended halflife factor concentrates. Haemophilia. 2017;23:566-574.

66. Wang L, Takabe K, Bidlingmaier SM, Ill CR,
Verma IM. Sustained correction of bleeding disorder in hemophilia B mice by gene therapy. Proc Natl Acad Sci U S A. 1999;96: 39063910

67. Chao H, Mao L, Bruce AT, Walsh CE. Sustained expression of human factor VIII in mice using a parvovirus based vector. Blood. 2000;95:1594 1599.

68. Mount JD, Herzog RW, Tillson DM, et al Sustained phenotypic correction of hemophilia B dogs with a factor IX null mutation by liver directed gene therapy. Blood. 2002;99:2670 2676

69. Sarkar R, Mucci M, Addya S, et al Long term efficacy of adeno associated virus serotypes 8 and 9 in hemophilia A dogs and mice. Hum Gene Ther. 2006;17:427 439.

70. Snyder RO, Miao C, Meuse L, et al. Correction of hemophilia B in canine and murine models using recombinant adeno associated viral vectors. Nat Med. 1999:5:64 70.

71. Nathwani AC, Tuddenham E, Rangarajan S, et al. Adenovirus associated virus vector mediated gene transfer in hemophilia B. N Engl J Med. 2011;365:2357 2365.

72. Nathwani AC, Reiss UM, Tuddenham E, et al. Long term safety and efficacy of factor IX gene therapy in hemophilia B. N Engl J Med. 2014:371:1994 2004.

73. Peyvandi F, Garagiola I. Clinical advances in gene therapy updates on clinical trials of gene therapy in haemophilia. Haemophilia. 2019;25(5):738-746

74. George LA, Sullivan SK, Giermasz A, et al. Hemophilia B gene ther apy with a high specific activity factor IX variant. N Engl J Med. 2017;377:2215 2227.

75. http://ir.sparktx.com/news releases/newsrelease details/spark therapeutics and pfizer announce data 15 participants.

76. Chapin J, Rottensteiner $\mathrm{H}$, Scheiflinger Monahan PE. An analysis of bleeding rates and factor IX consumption in the phase I/II BAX 335 gene therapy trial in subjects with hemophilia B. Abstracts of the XXVI Congress of the International Society on Thrombosis and Haemostasis. Res Pract
Thromb Haemost. 2017;1(Suppl. 1):144

77. Miesbach W, Meijer K, Coppens M, et al. Gene therapy with adenoassociated virus vector 5 human factor IX in adults with hemophilia B. Blood. 2018;131:1022 1031.

78. Leebeek F, Meijer K, Coppens M, et al Reduction in annualized bleeding and factor IX consumption up to 2.5 years in adults with severe or moderate severe hemophilia $\mathrm{B}$ treated with AMT 060 (AAV5 hFIX) gene therapy. Blood. 2018;132 (Suppl. 1): 3476.

79. Ward NJ, Buckley S, Waddington SN, et al. Codon optimization of human factor VIII cDNAs leads to high level expression. Blood. 2011;117:798 807

80. Siner JI, Iacobelli NP, Sabatino DE, et al Minimal modification in the factor VII $\mathrm{B}$ domain sequence ameliorates the murine hemophilia A phenotype. Blood. 2013;121: 43964403.

81. Rangarajan S, Walsh L, Lester W, et al AAV5 Factor VIII gene transfer in severe hemophilia A. N Engl J Med. 2017;377: 25192530

82. Pasi KJ, Rangarajan S, Mitchell N, et al. Multiyear Follow-up of AAV5-hFVIII-SQ gene therapy for hemophilia A. N Engl J Med. 2020;382:29-40

83. Donsante A, Miller DG, Li Y, et al. AAV vector integration sites in mouse hepatocellular carcinoma. Science. 2007;317(5837):477.

84. Nault JC, Datta $S$, Imbeaud $S$, et al. Recurrent AAV2-related insertional mutagenesis in human hepatocellular carcinomas. Nat Genet. 2015;47:1187-1193.

85. Manno CS, Pierce GF, Arruda VR, et al Successful transduction of liver in hemophilia by AAV-Factor IX and limitations imposed by the host immune response. Nat Med. 2006;12:342-347.

86. Kaeppel C, Beattie SG, Fronza R, et al. A largely random $\mathrm{AAV}$ integration profile after LPLD gene therapy. Nat Med. 2013;19:889891

87. D'Avola D, López-Franco E, Sangro B, et al Phase I open label liver-directed gene therapy clinical trial for acute intermittent porphyria. J Hepatol. 2016;65(4):776-783. 